Revue d'histoire de l'Amérique française

ZAS REVUE D.HISTOIRE DE L'AMÉRIQUE FRANÇAISE

\title{
En guise de supplément au Laurendeau de Monière
}

\section{Yves Laurendeau}

Volume 38, numéro 1, été 1984

URI : https://id.erudit.org/iderudit/304238ar

DOI : https://doi.org/10.7202/304238ar

Aller au sommaire du numéro

Éditeur(s)

Institut d'histoire de l'Amérique française

ISSN

0035-2357 (imprimé)

1492-1383 (numérique)

Découvrir la revue

Citer cette note

Laurendeau, Y. (1984). En guise de supplément au Laurendeau de Monière.

Revue d'histoire de l'Amérique française, 38(1), 73-89.

https://doi.org/10.7202/304238ar d'utilisation que vous pouvez consulter en ligne.

https://apropos.erudit.org/fr/usagers/politique-dutilisation/ 


\section{NOTE CRITIQUE}

\section{EN GUISE DE SUPPLÉMENT AU LAURENDEAU DE MONIËRE ${ }^{1}$}

YVES LAURENDEAU

Jusqu'à présent, pour connaître l'action et la pensée d'André Laurendeau, on n'avait facilement accès, en français, qu'à des portraits généralement chaleureux mais très rapides ${ }^{2}$, à deux études savantes mais n'embrassant chacune que deux moments et deux aspects limités de sa carrière ${ }^{3}$, au récit qu'il avait fait lui-même de la crise de la conscription $^{4}$, et à un recueil de chroniques rédigées pour le Magazine Maclean de 1961 à $1966^{5}$.

D'ailleurs, comment effectuer la synthèse d'un itinéraire en apparence aussi capricieux, d'une oeuvre aussi diverse, littéralement éparpillée aux quatre vents? Rien qu'entre 1947 et 1963, André Laurendeau aura été rédacteur au Devoir, et, on l'a dit, historien de la conscription et chroniqueur pour le Maclean; mais il aura aussi été directeur de l'Action nationale, commentateur ou animateur de plusieurs émissions à la radio ( $\mathrm{CBF}$ et $\mathrm{CBC})$ et à la télévision $(\mathrm{CBFT})$, romancier, dramaturge, adaptateur, conférencier, critique de disques, membre du Conseil provincial des arts... et j'en oublie.

M. Denis Monière a parcouru la plus grande partie de tout cela sans s'y perdre; et il nous en présente un tableau cohérent et raisonnablement complet dans son étude, André Laurendeau et le destin d'un peuple. Il offre une vue en perspective des morceaux de vie un peu épars qu'on connaissait, situant chacun dans une trame qui permet d'en saisir le sens. Voilà le principal mérite de $M$. Monière: nous montrer l'évolution de cet homme dont tant de ses cadets disent avoir trouvé en lui un modèle et une inspiration, et dont le cheminement intellectuel

\footnotetext{
1 Denis Monière, André Laurendeau et le destin d' un peuple (Montréal, Québec/Amérique, 1983)

Les plus récents ont été brossés par Simonne Monet Chartrand, Ma vie comme rivière, tome 2 (1939-1949) (Montréal, Éditions du remue-ménage, 1982), et par Gérard Pelletier, Les années d'impatience. 1950-1960 (Montréal, Stanké, 1983).

3 Gilles Durand, «La pensée socio-économique d'André Laurendeau», in Robert Comeau (éd.), Économie québécoise (Sillery, P.U.Q., 1969), 485-495, et Paul-André Comeau, Le Bloc populaire (Montréal, Québec/Amérique, 1982).

4 André Laurendeau, La crise de la conscription. 1942 (Montréal, Éditions du Jour, 1962). André Laurendeau, Ces choses qui nous arrivent (Montréal, HMH, 1970).
} 
fait la transition entre le nationalisme traditionaliste des années 1920 et la Révolution tranquille.

M. Monière ne s'étend guère sur les sources du nationalisme de Laurendeau; l'influence de Lionel Groulx est notée, mais pas suffisamment celle d'Henri Bourassa. Quant aux origines autochtones de ses idées d'ordre socio-économique, il y aurait eu lieu d'explorer davantage du côté d'un Édouard Montpetit, d'un Esdras Minville, ou même de l'Action libérale nationale. En revanche, sur l'influence de ses parents, sur celle du père Georges-Henri d'Auteuil, sur les préoccupations des étudiants de sa génération et de son milieu, sur son séjour en France qui le mit en contact avec une pensée catholique qui ne fût pas synonyme de conservatisme et qui ouvrit considérablement ses horizons, $M$. Monière est le premier à apporter des éclaircissements qui rendent beaucoup mieux compréhensible la suite de sa carrière. De cette suite, il présente une synthèse qui, par son ampleur, dépasse très largement tout ce qu'on a publié jusqu'à présent sur Laurendeau.

\section{Alors, pourquoi ce «Supplément»?}

Parce que je suis le troisième des six enfants d'André Laurendeau (1912-1968) et de Ghislaine Perrault (1914-1976). Voilà mon père transformé sous mes yeux en objet d'étude. Je le reconnais assez dans le portrait qu'en trace patiemment $\mathbf{M}$. Monière. Mais je crois qu'il y manque un ou deux éléments importants. En outre, dans quelques traits, je ne sais trop si je ne reconnais pas mieux $M$. Monière que mon père. Avant que ce dernier ne m'échappe tout à fait, devenu statue au Musée de l'histoire, ou pièce à conviction dans quelque débat constitutionnel, je veux m'assurer que la copie se conforme le plus exactement possible à l'original tel que je l'ai connu.

Je n'entends pas ici récuser, ni récrire, mais uniquement retoucher et compléter un ouvrage que je crois solide dans son architecture d'ensemble. Qu'on ne prenne mon article que pour ce qu'il est, c'est-à-dire un ensemble quelque peu décousu de réflexions et de remarques sur le Laurendeau de Denis Monière.

\section{L'agnosticisme d'André Laurendeau}

Dans cette étude consacrée tout entière à la pensée d'André Laurendeau et à son évolution, M. Monière a choisi, Dieu sait pourquoi, de taire son agnosticisme. Une telle omission, étonnante en soi ${ }^{6}$, me paraît d'autant moins admissible que $\mathrm{M}$. Monière a pris la peine, dans ses chapitres III et IV, d'analyser divers aspects du catholicisme de Laurendeau durant les années 1930.

\footnotetext{
$6 \quad$ Trois des personnes que M. Monière a interviewées et qu'il remercie au début de son livre lui ont parlé de manière circonstanciée de l'agnosticisme d'André Laurendeau, en juin et en août 1983.
} 
André Laurendeau a perdu la foi dès les années 1940. Mis à part les confesseurs, seule sa femme l'a su à cette époque. Plus tard, il s'en est confié à Charlotte Boisjoli. En 1964 et 1965, enfin, il l'a dit ou écrit à ses quatre aînés. Peut-être d'autres personnes ont-elles reçu cette confidence, mais cela semble assez peu probable.

Officiellement, extérieurement, Laurendeau a toujours tenu à se montrer croyant et pratiquant. Pourtant, il n'allait pas à la messe: cela pouvait être mis sur le compte de son agoraphobie, alibi idéal car elle n'était pas simulée, seulement un peu exagérée pour les besoins de la cause.

Sur les circonstances de la perte de la foi, sur les motifs de son silence, il s'est expliqué par écrit par deux fois, en 1964. D'abord, à mots couverts, dans le Magazine Maclean ${ }^{7}$; puis, en termes clairs, dans une lettre à son fils Jean ${ }^{8}$.

Dans sa lettre, il écrivait:

Donc j'ai perdu la foi, et si je puis mettre une date sur cet aveu, c'est grâce à toi, qui ne t'en doutais guère. Tu as fait ta première communion le 19 mai 1945 . D'habitude, à cette cérémonie, les parents communient eux aussi: je l'avais fait pour Francine deux ans plus tôt. Mais je venais de m'avouer que je ne le pouvais plus. Je me suis donc fait porter malade, et $j$ 'ai passé la journée dans mon lit — furieux de devoir jouer cette comédie. Je m'y croyais forcé: j'étais chef provincial d'un parti officiellement catholique, je siégeais comme député en face de Duplessis, dont le règne commençait. J'avais trente-trois ans. Je me débattais «contre le doute» depuis au moins sept ans, peut-être depuis dix ans (1935, mon arrivée à Paris), peut-être depuis toujours: il me semble que j'ai toujours oscillé de l'incroyance au fanatisme [religieux]. Mais en cette année 1945, il a fallu que je me rende, que je m'admette à moi-même: il n'y a plus rien, c'est fini.

Il y avait eu deux sortes de motifs. Les uns étaient de type rationnel: «la non-universalité de l'Église après vingt siècles, le fait qu'on attrappe sa foi avec sa race, par le hasard de la naissance»; des objections, aussi, «sur l'historicité du Christ et la valeur mythique de son personnage». Les autres motifs étaient de type plus empirique; en particulier la sensation que, quand on prie, on ne fait que se parler à soimême.

Dans ce temps-là, ton confesseur te dit: «C'est une tentation.» Mais il me semble que ce sont les parties les plus nobles de mon être qui sont engagées. «Orgueil». Merde au confesseur.

\footnotetext{
7 Le magazine Maclean, mai et juin 1964. Textes reproduits in Ces choses qui nous arrivent, 192-201.

8 Lettre à Jean Laurendeau, juillet 1964. Ce document ne se trouve pas à la Fondation Lionel-Groulx, mais on avait offert à $\mathrm{M}$. Monière de le lui montrer.
} 
Et on s'avoue enfin qu'on ne croit plus.

Notons qu'André Laurendeau ne fera jamais profession d'athéisme. Il jugeait que le terme d'agnosticisme correspondait mieux à l'absence de certitude où il se trouvait quant à la non-existence ou à l'existence d'un Dieu.

Une fois l'aveu fait à soi-même, puis à l'épouse, se pose le problème de l'attitude à adopter face aux enfants. Mais

comment vous confier ce que vous n'auriez pas compris et [qui] vous aurait troublés? J'ai pris l'habitude du silence. J'ai failli la rompre huit ans plus tard: en 1953, tu avais 15 ans, Francine 17, je me sentais faux devant vous, devais-je vous parler? [Ta mère] et moi avons convenu que je prendrais le conseil d'un tiers: ce fut le P. Régis ${ }^{9}$, dont j'avais aimé le dernier carême à l'École des Parents. Il n'a pas pris mon incroyance au sérieux, l'assimilant à une névrose, et il a opté pour le silence. J'ai donc continué de me taire. Au reste, j'avais peur d'une indiscrétion de jeune homme ou de jeune fille, et, socialement, $j^{\prime}$ avais besoin $d u$ secret ${ }^{10}$, car j'étais journaliste à un quotidien catholique.

Ceci me posait un problème. Je me disais: ai-je le droit? le lecteur me prend pour ce que je ne suis pas. [...] J'en suis encore là: le secret m'est toujours socialement nécessaire, et je le trouve honteux, beaucoup moins cependant que jadis; $j$ 'en ai pris mon parti. [...]

J'ai $[\ldots]$ ressenti d'abord la perte de la foi comme un manque, un vide, ou même comme une infirmité. Je n'avais pas l'agnosticisme conquérant, mais honteux. Je n'aurais pas eu l'idée de m'en glorifier, mais de l'avouer, si je l'avais cru possible. Aussi me suis-je souvent reconnu dans La ligne du risque, de Pierre Vadeboncoeur. ${ }^{11}$

Ce genre de divorce d'avec son milieu et, en définitive, d'avec soi-même, André Laurendeau l'a évoqué dans sa chronique du Magazine Maclean, en la personne d'un marguillier de province «qui a trompé tous ses proches sur ce qu'il est» et qui s'en confesse à lui. Ce marguillier, bien évidemment, c'est Laurendeau lui-même, facilement reconnaissable sous le masque.

Quand [le marguillier] parle de sa foi perdue, du mensonge qu'il maintient auprès de tous, $j$ 'ai parfois la sensation d'être un confesseur, on dirait qu'il va me demander l'absolution. ${ }^{12}$

9 Louis-M. Régis, dominicain.

10 Souligné par Ancré Laurendeau.

11 Court essai de Pierre Vadeboncoeur paru dans Situations, 4e année, $\mathrm{n}^{\circ}$ 1, [1962]: 1-58 (reproduit, avec une ou deux retouches, in La ligne du risque. Essais (Montréal, HMH, 1963) 165-218).

2 André Laurendeau, Ces choses..., 197. 
Pour le marguillier, révéler son incroyance aux siens qui n'y eussent rien compris, c'eût été rompre avec eux, devoir refaire sa vie ailleurs.

- Qu'auriez-vous fait à ma place? Je suis un brave homme d'athée, pas un héros... ${ }^{13}$

Alors, il restait le silence, avec le sentiment de culpabilité, l'étouffement, la sclérose...

Le péché du Marguillier, à ses propres yeux, ce n'était point de ne pas croire, mais de présenter à ses concitoyens une image fausse de lui-même. Il fallait enfin que quelqu'un sût qui il était, et le hasard m'a imposé d'être ce témoin.

L'intolérance de son milieu était-elle aussi redoutable qu'il le croyait? Il a sans doute eu tort, d'abord pour lui-même, de se cacher, mais non de craindre le scandale et la vengeance. [...] Le Marguillier, en tant que non-conformiste, aurait été vomi par toutes les communautés étroites et homogènes: son chef-lieu ne fait pas tache, il est l'incarnation même des lois que vit une société fermée sur elle-même. ${ }^{14}$

Enfin, l'auteur disait écrire ce texte

en mémoire d'un pauvre diable englué dans ce marécage et qui n'a jamais réussi à se libérer - d'un notable de province entouré de l'estime de ses concitoyens parce qu'il n'avait pas contrevenu à leurs lois, s'étant au préalable soigneusement avorté lui-même. ${ }^{15}$

Comme M. Monière, je considère qu'Une vie d'enfer et Deux femmes terribles, les oeuvres littéraires les plus désespérées d'André Laurendeau, sont l'expression d'une sorte de non-être canadien-français, ainsi qu'un exutoire aux sentiments de culpabilité résultant d'une liaison amoureuse. Mais comment ne pas voir que l'instinct «d'autodestruction» (Monière, p. 246 et 255), la volonté de vous montrer pire que ce qu'on vous croit, l'insistance sur l' «hiatus entre les apparences et la réalité des êtres» (p. 257), y sont aussi l'expression à peine transposée de la culpabilité plus ancienne que lui faisait éprouver le secret de son incroyance, et, peut-être, les premiers pas en direction d'un aveu encore jugé impossible? M. Monière s'est privé là, à n'en pas douter, d'une clef importante pour comprendre l'évolution de Laurendeau et pour interpréter certains aspects de son oeuvre.

S'avancer, oui, mais sans devancer trop. N'étant pas armé des certitudes qui créent les prophètes, André Laurendeau n'a pas voulu la rupture. Plus qu'un Paul-Émile Borduas ou qu'un François Hertel, il a craint l'opprobe des siens. Il a préféré, à l'ostracisme ou à la stérilité

\footnotetext{
3 Ces choses..., 198.

14 Ces choses..., 200.

15 Ces choses..., 201.
} 
d'un exil intérieur, l'assurance de pouvoir être entendu dans son milieu, de la tribune où il se trouvait déjà. Mais de quels déchirements, de quel mépris de soi n'a-t-il pas payé ce choix, avant de trouver, dans la cinquantaine, un début de réconciliation avec lui-même?

\section{Comment devient-on André Laurendeau?}

Il me paraît profondément juste de dire que «chez cet intellectuel, la circulation entre l'individuel et le collectif est particulièrement intense» (p. 14). Mais je ne puis me défendre de l'impression qu'un peu trop souvent, quand il s'agit d'expliquer les orientations ou les préoccupations d'André Laurendeau, M. Monière privilégie le pôle collectif, où il se sent plus à son aise et qui inspire davantage en lui l'historien et le politicologue, au détriment du pôle individuel, qui, je puis en témoigner, a compté pour beaucoup.

Cela n'est nulle part aussi apparent que dans le chapitre $\mathrm{X}$, consacré à l'oeuvre littéraire de Laurendeau. C'est le seul chapitre - indépendamment de la question de l'agnosticisme - qu'il faille corriger substantiellement. D'abord parce que les erreurs y sont peut-être plus importantes qu'ailleurs. (J'y reviendrai plus loin.) Ensuite, et surtout, parce que la lecture que fait $M$. Monière des oeuvres littéraires de mon père, quoique légitime, reste partielle.

Qu'on me comprenne bien. Voir dans la création littéraire un «refuge contre le désespoir» (p. 237), expliquer le thème de la révolte contre la mère par la «révolte contre une religion sans âme, contre une Église omniprésente» (p. 249), ou expliquer le sentiment d'échec, d'impossibilité à être, par «les stigmates de la colonisation très profondément inscrits en [Laurendeau]» (p. 257), voilà autant d'interprétations où je n'ai pas de difficulté à suivre M. Monière. Mais pourquoi la création littéraire était-elle si nécessaire chez Laurendeau, et pourquoi ces sentiments avaient-ils de telles résonances en lui? Sur ce genre d'interrogations, M. Monière passe presque toujours trop vite. Indiquons quelques pistes.

D'abord, il faut souligner que le milieu familial d'André Laurendeau était plus exceptionnel encore que ne l'a dit $\mathbf{M}$. Monière. Ses parents, Arthur Laurendeau (1880-1963) et Blanche Hardy (1880-1957), avaient une grande curiosité artistique et intellectuelle. Mais on a des indices que cette attitude était partagée, à l'époque, par leurs familles respectives. Le père de Blanche, Edmond Hardy, marchand d'instruments et de partitions de musique, directeur de l'Harmonie de Montréal, occasionnellement éditeur, était bien placé pour se tenir en contact avec les principaux courants musicaux. La tradition familiale veut que ce soit lui (à moins que je ne confonde avec son père, qui avait été lui aussi directeur de fanfare) qui ait été le premier, chez nous, à jouer du saxophone et à faire connaître cet instrument. Du côté des Laurendeau, 
il faut au moins signaler que le frère aîné d'Arthur, Albert, médecin à Saint-Gabriel-de-Brandon, publia en 1911 un livre sur la théorie de l'évolution ${ }^{16}$. Non content de s'en faire l'apôtre, il osait - tout en proclamant sa foi catholique - revendiquer «les droits de la science», déplorer l'ignorance de ses confrères médecins et de trop d'évêques, et dénoncer au passage l'enseignement désuet prodigué par les collèges classiques. C'était trop au gré de Mgr Joseph-Alfred Archambault, premier évêque de Joliette, qui mit l'ouvrage à l'Index et condamna l'auteur.

Un milieu, donc, et une époque d'orthodoxie rigoureuse, mais que des idées, des nouveautés, arrivaient tant bien que mal à pénétrer. Il a fallu, certes, de l'audace au jeune Arthur Laurendeau, dans les années 1900, pour abandonner ses études de droit et se lancer dans une carrière de chanteur. Et je parie qu'on pourrait compter sur les doigts d'une seule main les Canadiennes françaises (et peut-être les Canadiennes tout court) qui, comme Blanche Hardy, sont devenues au début du siècle des pianistes professionnelles. Mais sans doute leurs familles les avaientelles prédisposés plus qu'on ne croit à ces «audaces».

Un facteur plus directement marquant dans la formation d'André Laurendeau fut le tempérament de sa mère. Edmond Hardy avait eu un fils et trois filles. D'Edmond, André Laurendeau a toujours parlé comme d'un faible et d'un aimable raté; de son fils, comme d'un raté minable. L'aînée des filles, Hermine, fut abandonnée par son mari après quelques mois de mariage et n'en entendit plus parler. Elle mourut nonagénaire, pour ainsi dire vieille fille, un unique projet de remariage ayant échoué parce que l'autorité religieuse avait exigé des preuves, évidemment impossibles à fournir, de la mort du disparu. Anna, l'autre soeur de Blanche, eut une vie matrimoniale plus agitée. Elle quitta son mari pour un Belge avec qui elle vécut en concubinage ouvert pendant un certain temps. Mais elle fut à son tour abandonnée par son amant, et elle finit par réintégrer, penaude mais sans prononcer un mot d'excuses, le foyer conjugal.

Dans la famille d'André Laurendeau, la branche maternelle avait donc de fortes aptitudes au malheur. S'étonnera-t-on, avec cela, que Blanche, femme entière et passionnée, ait été toute sa vie tourmentée et inquiète? Dès l'adolescence, elle fut affligée d'un asthme chronique qui amplifiait ses angoisses et la tenailla jusqu'à la mort. Sa bellefamille voyait en elle une pianiste accompagnatrice remarquable, mais pour le reste, la jugeait emportée ou même fantasque. Peu avant son mariage, elle reçut un coup dont elle fut certainement profondément ébranlée. Arthur Laurendeau, avec qui elle était vaguement engagée depuis 1906, avait passé toute l'année 1908 à Paris, pour y étudier le

16 Albert Laurendeau, La vie. Considérations biologiques (Saint-Gabriel-de-Brandon, 1911). 
chant. À son retour, il alla loger à Westmount chez son frère Rémus, qui était alors au sommet d'une fortune assez considérable réalisée dans la spéculation foncière. Blanche et Arthur se fiancèrent. Mais une jeune Française, débarquée sans crier gare, retraça Arthur chez Rémus, et alla l'y accuser non seulement de l'avoir plaquée, mais encore d'être le père du bébé qu'elle avait amené avec elle. Arthur ne nia rien. Elle menaça d'empêcher le mariage prévu par un procès ou par un scandale public durant la cérémonie. Il semble qu'elle n'eut pas à s'exécuter: Rémus lui donna une somme considérable (cinq mille dollars, dit-on). Était-ce ce qu'elle était venue chercher? Elle reprit le bateau avec l'enfant, et on perdit sa trace - qu'à vrai dire on ne tenait pas à retrouver ${ }^{17}$. Entre Blanche et Arthur, les choses se raccommodèrent tant bien que mal; plutôt bien que mal, je suppose, puisque le mariage eut lieu, le 7 février 1910. Mais Blanche resta marquée. Déjà tourmentée, elle devint jalouse, possessive... et de plus en plus asthmatique.

Elle aurait voulu plusieurs enfants, mais n'eut qu'un fils, sur qui ses soeurs et elle reportèrent une partie de leurs frustrations sentimentales. André Laurendeau a été, certes, «surprotégé» (p. 17); mais sa «santé fragile» (p. 17 et 28) eut assez peu à y voir. À quoi j'ajouterai que tous les fils uniques ne sont pas forcément chéris par deux tantes maternelles restées sans enfants. Il n'est pas fréquent, non plus, que leur mère fasse sculpter leur buste par un Elzéar Soucy (à onze ans!), ni qu'elle leur impose des cours de ballet (André Laurendeau en éprouva une humiliation si grande qu'il le dissimulera comme une tare auprès des copains, et encore plus soigneusement une fois devenu homme public). André Laurendeau confiera, beaucoup plus tard, que durant le séjour de deux ans en France qui suivit son mariage, sa mère lui avait trop souvent écrit qu'elle s'ennuyait de lui,

au point par moments de m'empoisonner l'Europe. («Cette pauvre femme vieillissante et malade dont je suis le seul enfant...») ${ }^{18}$

À partir de son retour au Québec, sa femme, ses enfants et lui passèrent toutes leurs vacances en compagnie de Blanche, dans la maison d'été qu'elle avait achetée en 1920 à Saint-Gabriel-de-Brandon; cela dura jusqu'en 1949, date après laquelle son piètre état de santé lui interdit de quitter Montréal.

Le rapport mère ombrageuse / fils unique a certainement compté pour beaucoup plus que ne le dit $\mathbf{M}$. Monière dans la formation du caractère d'André Laurendeau. On peut y voir une cause importante de la prédisposition au secret, de la culpabilité latente, de la sensation

\footnotetext{
17 Il existe d'autres versions, plus théâtrales mais moins sûres, de cette histoire. Je m'en suis tenu ici aux souvenirs de Mlle Marguerite Laurendeau, fille de Rémus et nièce d'Arthur, et de M. Jean Lallemand, lui aussi neveu d'Arthur par sa mère Albertina Laurendeau.

is Lettre à Jean Laurendeau, juillet 1964.
} 
d'étouffement qui ont souvent marqué sa vie - nous l'avons vu à propos de son agnosticisme - et dont ses oeuvres littéraires se font l'écho. Cela n'explique pas tout, loin de là. Mais tous ceux qui ont vécu une situation «coloniale», ou (p. 249) «l'oppression cléricale», n'ont pas forcément écrit ce qu'ont écrit un André Laurendeau ou un Saint-Denys Garneau. Il me semble qu'ici encore M. Monière s'est satisfait d'explications partielles.

André Laurendeau a évoqué, lors de sa réception à la Société royale du Canada, son enfance baignée de musique. Il gardait l'impression, dans l'âge mûr, que celle-ci le nourrissait davantage encore que la littérature ${ }^{19}$. Mais il a dit aussi combien cette dernière avait imprégné ses rapports avec ses parents.

Mon père ne savait pas toujours parler aux enfants; il les traitait volontiers comme des grandes personnes; c'est ainsi qu'il me fit lire à onze ans l'Iliade dans la traduction de Leconte de Lisle, qui du reste me conquit, ainsi que le $\mathrm{Cid}$, dont la vaillance m'exalta. Un peu plus tard je pris en horreur Mademoiselle de Lespinasse, dont ma mère dévorait les lettres d'amour, et qu'elle discuta à table avec son mari durant d'interminables semaines, suivies de mois consacrés à Balzac. ${ }^{20}$

Arthur lui parlait souvent de Phèdre, qu'il avait vu jouer par Sarah Bernhardt à Paris. Il lui communiqua très jeune son enthousiasme pour Péguy, qu'il lisait fort bien à voix haute.

«C'est pas possible d'avoir élevé un enfant comme ça!», blaguait Gérard Filion ${ }^{21}$.

Fils unique d'un père dont la voix sonore, la prestance, le verbe généreux, faisaient un modèle difficile à égaler, et d'une mère exigeante et possessive qui, de surcroît, plaçait en lui d'immenses espoirs, Laurendeau, au sortir de cette enfance trop sérieuse et d'une dépression qui dura deux ans, finit par opter pour l'engagement social et politique, alors que tout jusque-là paraissait le vouer à une carrière littéraire ou musicale. Pourquoi?

Affaire de circonstances et de climat social, comme le montre avec raison M. Monière (notamment p. 48-55). Mais aussi, problèmes que lui posaient sa sensibilité très vive et sa difficulté à se donner une identité face à de tels parents. La création littéraire ou musicale comportait des risques trop grands pour un André Laurendeau encore peu sûr de

\footnotetext{
19 «Réponse de M. André Laurendeau de la Société royale du Canada», in Présentation, $\mathrm{n}^{\circ}$ 19, Société royale du Canada, section française, année académique 1964-1965: 19-26 (reproduit in Écrits du Canada français, ${ }^{\circ} 35$ (1972): 57-64). Voir aussi Francine Laurendeau, «Mon père, ce héros au sourire si doux", in L'incunable (Bulletin de la Bibliothèque nationale du Québec), $18^{\mathrm{e}}$ année, $\mathrm{n}^{\circ} 1$ (mars 1984): 11-15.

20 «Réponse...», 22.

21 D’après Pierre Laporte, «Laurendeau, journaliste, député», in Le Devoir, 3 juin 1968.
} 
lui. À cet égard, le titre du livre de $\mathrm{M}$. Monière me paraît judicieusement choisi. André Laurendeau, en effet, s'est très tôt et très étroitement identifié au destin de son peuple. Dès l'âge de vingt ans, cela a orienté presque toute sa carrière. Ne pourrait-on y voir l'effet de la nécessité, pour lui dont l'identité était fragile, de se reposer sur plus grand, sur plus fort que soi?

Il faut probablement y voir en outre une sorte de réflexe de santé, comme l'a noté avec beaucoup de perspicacité Fernand Dumont, qui n'était pourtant pas un intime de Laurendeau:

Cet homme s'était sûrement voué aux débats historiques pour se guérir chaque jour d'une vive sensibilité qui, trop tournée vers l'intérieur, se serait perdue dans sa propre mort. Les enfants frêles et émotifs sentent parfois qu'ils doivent devenir botaniste ou journaliste pour écarter de soi la corrosion du regard. Laurendeau rappelait souvent la ferveur de son enfance pour la musique; il parlait admirablement de Debussy, mais avec un accent qui, pour moi en tout cas, montrait de toute évidence qu'il avait été littéralement sauvé en ne prenant pas cette voie. ${ }^{22}$

Quand, à partir de son retrait de la politique active, il fut de plus en plus tenté de revenir à la voie des arts et de la création, ce n'était pas seulement «parce que l'horizon politique était bouché» (p. 249), ni nécessairement parce que «l'imaginaire était le meilleur refuge contre le désespoir» (p. 237), mais certainement "pour réaliser un rêve de jeunesse» (p. 237) dont les nécessités de sa vie intérieure comme les circonstances extérieures l'avaient d'abord détourné.

Comment devient-on André Laurendeau?

À cela les réponses sont multiples, peut-être inachevables. Je ne veux pas invalider celles que propose M. Monière. Mais il m'a semblé qu'il fallait les compléter par d'autres, que je viens d'esquisser sans prétendre avoir épuisé le sujet.

\section{Où le politicologue a raison malgré le polémiste}

M. Monière ne cache pas ses options politiques: il est un indépendantiste avoué. Ses références sont connues; il a lu Marcel Rioux ${ }^{23}$, Gilles Bourque ${ }^{24}$. Son interprétation de l'oeuvre littéraire de mon père doit sans doute quelque chose à Albert $\mathrm{Memmi}^{25}$. Rien de tout cela ne le rend inapte à analyser les aspects politiques de l'oeuvre de Laurendeau. Il le fait d'ailleurs avec une sympathie souvent perceptible. À

\footnotetext{
Fernand Dumont, Préface à Ces choses..., xvii.

Marcel Rioux, La question du Québec (éd. revue et augm., Montréal, Parti pris, 1976).

Gilles Bourque, Question nationale et classes sociales au Québec (1760-1840) (Montréal, Parti pris, 1970).

${ }_{25}$ Albert Memmi, Portrait du colonisé (éd. revue et corr., Montréal, L’Étincelle, 1972).
} 
quelques reprises, cependant, l'outrance des propos nous le fait soupçonner de s'intéresser moins à nous restituer André Laurendeau et son époque tels qu'ils furent, qu'à nous montrer combien l'indépendance du Québec est nécessaire.

Ainsi, je peux bien suivre $M$. Monière quand il voit dans l'antisémitisme canadien-français «un effet pervers du colonialisme» (p. 61). Mais je ne saisis pas du tout le sens, dans une page par ailleurs bien menée sur l'antisémitisme des Jeune-Canada, d'une phrase comme celleci:

Enfin dans les situations de domination coloniale, le colonisateur applique une stratégie de diversion qui a pour effet d'attiser les rivalités ethniques pour diviser les groupes dominés et les détourner des causes réelles de leur subordination (p. 61).

M. Monière voudrait-il signifier par là que les Jeune-Canada, en dénonçant la «juiverie internationale», étaient directement manipulés par $S t$. James Street ou Bay Street? L'hypothèse est tellement invraisemblable que je suppose que ce n'est pas ce qu'il a voulu dire. Mais alors, quel est donc le lien entre les événements de 1933 et cette remarque qui paraît tout droit sortie du De bello gallico?

Plus loin, je comprends mal $\mathrm{M}$. Monière d'en vouloir encore aux pères de Sainte-Croix pour avoir sapé la prédominance de l'A.C.J.C. par la création de la J.É.C., «apolitique et dégagée de toute préoccupation nationaliste», et qui «deviendra l'école de formation et de recrutement des cadres de la future élite politique fédéraliste de Cité Libre au gouvernement Trudeau» (p. 68-69). Jugement pour le moins surprenant, qui accorde une importance énorme à ces mouvements de jeunesse en tant qu'outils de formation intellectuelle et politique, et qui ne tient nul compte de ce que bon nombre de cadres et de militants du Bloc populaire, puis du R.I.N. et du Parti québécois, étaient passés eux aussi par les rangs de la J.É.C.

Quand M. Monière écrit que les nationalistes, lors du «plébiscite» de 1942, «avaient fait la preuve de l'iniquité du système fédéral et de l'impossibilité d'y faire respecter les intérêts et la volonté du Canada français» (p. 144), on ne peut s'empêcher, encore là, de trouver l'argumentation un peu courte. On aimerait plutôt que l'auteur explique un peu plus longuement pourquoi les fondateurs de la Ligue pour la défense du Canada - n'est-ce pas précisément l'objet de son étude? - n'ont pas conclu, quant à eux, à la nécessité de l'indépendance du Québec, et ont au contraire opté pour la création d'un parti fédéraliste, le Bloc populaire.

M. Monière nous lance carrément sur une fausse piste quand, pour expliquer les résultats des élections provinciales de 1944, il affirme que «le réflexe conservateur eut raison des espoirs nationalistes et ramena 
l'Union nationale au pouvoir avec une majorité absolue de [quarantehuit] sièges à l'Assemblée législative» (p. 164). Ignore-t-il que l'Union nationale a recueilli, en 1944 , moins de suffrages que les libéraux, et même, un pourcentage des suffrages exprimés légèrement moins élevé que celui, déjà faible (39\%), qu'elle avait obtenu aux élections précédentes et qui lui avait alors fait perdre le pouvoir? Maurice Duplessis et son parti regagnèrent le pouvoir en 1944 grâce à la conjonction extraordinaire de trois facteurs qui n'ont pas grand-chose à voir avec le conservatisme éventuel de l'électorat: une carte électorale qui donnait au vote rural un poids démesuré, le mode de scrutin uninominal à un tour, et la présence d'un tiers parti assez fort pour «diviser le vote».

Il paraît que si les élections fédérales de 1963 ont permis aux libéraux de former un gouvernement minoritaire, c'est «grâce au changement d'allégeance des Québécois, qui étaient revenus au bercail libéral attirés par les promesses de réformes constitutionnelles» (p. 280). Eston si sûr que de telles promesses ont alors été le facteur électoral déterminant, au Québec? De toute façon, $M$. Monière confond allègrement moins de $46 \%$ des suffrages exprimés avec «les Québécois»; et il ne tient pas compte de ce que dans le reste du Canada aussi, pas seulement au Québec, les libéraux avaient obtenu une majorité relative des suffrages.

Jusqu'ici, en somme, rien de bien grave, mais des travers agaçants dans un ouvrage par ailleurs si fouillé. Arrivons au chapitre XIII, consacré aux dernières années de la vie de Laurendeau et au bilan de sa participation à la Commission royale d'enquête sur le bilinguisme et le biculturalisme.

M. Monière, évoquant le fait que la plupart des groupes qui, au Québec, avaient comparu devant la Commission réclamaient des changements constitutionnels, souligne que le nouveau statut réclamé prenait, «pour beaucoup, la forme des Etats associés», et cite à ce propos (p. 321) le rapport préliminaire de la Commission. Pourtant, ce n'est qu'une parmi les formules proposées dont le rapport fait état; on n'y trouve même pas que celle qu'a retenue $\mathbf{M}$. Monière ait eu plus d'adeptes que les autres ${ }^{26}$. Ici, sans nous en aviser, l'auteur s'intéresse plus aux origines du Parti québécois qu'à son sujet déclaré.

Quand M. Monière, qui déplore «la tradition apolitique du nationalisme canadien-français», écrit qu' «on ne peut faire fi de la dynamique du pouvoir et des jeux de l'arène électorale», et lance presque à l'intellectuel une invitation à descendre «sur la place publique pour assumer lui-même le pouvoir politique» (p. 341), on croit bien comprendre les raisons de son engagement dans le comité directeur de la

\footnotetext{
26 Rapport préliminaire de la Commission royale d'enquête sur le bilinguisme et le biculturalisme (Ottawa, Imprimeur de la Reine, 1965), 83-85.
} 
Ligue d'action nationale ou dans le bureau national du Parti nationaliste. Il n'est pas évident pour autant que le chapeau coiffe André Laurendeau, ancien politicien lui-même mais qui avait éprouvé jusque dans sa chair que son tempérament ne s'accordait pas avec les jeux de l'arène électorale, et qui, comme éditorialiste, n'a jamais fait fi de la dynamique du pouvoir.

$M$. Monière expose les deux conceptions du rapport final - et des relations entre le Québec et le reste du Canada - qui s'affrontaient au sein de la Commission. Selon la première, dite verticale, et à laquelle se rattachait Laurendeau, le général devait précéder le particulier, ce qui impliquait qu'on devait d'abord s'entendre sur «la question du rapport entre le Québec et le reste du Canada» (p. 331). L'autre conception, dite horizontale, majoritaire celle-là, «consistait à présenter séparément chacun des aspects du problème», et à «ne présenter qu'à la fin les principes, les concepts et les recommandations générales»; ceux qui favorisaient cette approche insistaient «sur le rôle protecteur que le gouvernement fédéral devait jouer envers les minorités» (p. 331-332). Le triomphe de ce dernier point de vue, selon $\mathrm{M}$. Monière qui invoque ici le témoignage de Léon Dion, «fut la grande défaite de Laurendeau». Car ce dernier

savait qu'il serait impossible de revenir à la fin sur les concepts de dualité et d'égalité. [...] Il serait désormais impossible à la Commission de faire des recommandations sur les droits collectifs et sur la reconnaissance institutionnelle de l'existence de deux majorités. Cette orientation consommait l'échec de Laurendeau qui estimait que le statut politique du Québec était au coeur de la crise canadienne (p. 332).

On peut douter, en effet, que s'il eût vécu, Laurendeau fût parvenu à rallier à son point de vue une majorité des membres de la Commission; et il paraît encore moins sûr que le gouvernement Trudeau l'eût écouté sur ce point. Mais savait-il tout cela? Il me semble, en tout cas, qu'il n'avait pas renoncé à ses principaux objectifs, et qu'il conservait encore des espoirs à cet égard. M. Monière signale lui-même qu'il était entendu que le dernier livre du rapport devrait «aborder les grandes questions constitutionnelles relatives à l'avenir des deux sociétés» (p. 334 ${ }^{27}$. Et après tout, ce sont tous les commissaires qui ont signé l'Introduction générale en signant le premier livre. Or, cette introduction reconnaît «dans le Québec les principaux éléments d'une société francophone distincte» (p. 335); elle affirme nettement l'existence d'une dimension politique au problème de la coexistence au Canada de deux cultures dominantes ${ }^{28}$. D'ailleurs, l'auteur ne contredit-il pas lui-même son affirmation de la page 332 , quand il dit que

27 Ici, M. Monière paraphrase l'Avant-propos du Rapport de la Commission royale d' enquête sur le bilinguisme et le biculturalisme: (Ottawa, Imprimeur de la Reine, 1967), I: viii.

28 Rapport..., I: XXXV-XXXVIII. 
Laurendeau n'a jamais abandonné l'idée d'une réforme constitutionnelle qui consacrerait l'autonomie et l'extension des pouvoirs du Québec (p. 318)

et quand il soutient que, jusqu'à la fin,

malgré les frustrations et les déceptions éprouvées en cours de route, il continuait d'espérer que l'oeuvre entreprise débouche sur une réforme de la Constitution qui renforcerait l'autonomie du Québec (p. 345)?

Les quelques interprétations gauchies que j'ai tenté de redresser, ajoutées à une véhémence contre Pierre Elliott Trudeau qui, pour le moins, surprend, ne contribuent pas à éclairer le débat. Elles desservent tant la pensée de Laurendeau que le propos de $\mathrm{M}$. Monière. Je crains un peu que des lecteurs qui ne partageraient pas les convictions indépendantistes de l'auteur n'en concluent que celui-ci nous présente une image faussée des dernières années de Laurendeau. Rappelons-nous d'ailleurs qu'il y a trois ans, M. Jean Chrétien, alors ministre de la Justice fédérale, s'était cru autorisé à invoquer André Laurendeau à l'appui de la récente réforme constitutionnelle ${ }^{29}$. Dans un tel contexte, les mises au point n'auront quelque chance de convaincre que si elles paraissent inspirées de l'objectivité la plus limpide.

Or, tous les textes publiés, de même que le journal inédit de mon père, montrent que $M$. Chrétien se trompait absolument, et que $M$. Monière a presque toujours raison. Tout porte à croire, en effet, qu'André Laurendeau «n'aurait pas accepté, comme l'a fait son successeur [Jean-Louis Gagnon], le rétrécissement des objectifs de la Commission» (p. 344) (30 $^{30}$. Et je tiens pour clairement établi que les «thèses trudeauistes» (p. 343) sur le bilinguisme ont abouti à une «récupération centralisatrice des travaux de la Commission» (p. 344).

J'estime, en d'autres termes, que $M$. Monière s'est fait une représentation fidèle des idées et des espoirs d'ordre constitutionnel d'André Laurendeau à la fin de sa vie. Mais, en transmettant cette représentation, la voix du polémiste a couvert à l'occasion celle de l'historien et du politicologue. C'est pourquoi je souhaite qu'une deuxième édition permette d'éliminer ces interférences qui risquent bien inutilement de semer le doute sur la véracité et l'objectivité de l'ouvrage dans son ensemble.

Pour l'essentiel, je reconnais trois mérites à l'ouvrage de $\mathrm{M}$. Monière. Le premier, je l'ai déjà dit, c'est d'offrir une vue d'ensemble de la carrière d'André Laurendeau, vue dont l'équivalent n'existait

\footnotetext{
${ }^{29}$ Cf. Marcel Adam, «En cas de doute, testons la franchise», in La Presse, 19 février 1981, et Michel Roy, «D'André Laurendeau à Jean Chrétien», in Le Devoir, 19 février 1981 .

30 Point de vue partagé par Claude Ryan, "Le rêve trahi d'André Laurendeau», in Le Devoir, 11 mars 1971.
} 
encore nulle part. Le deuxième, c'est d'avoir balisé le terrain; même quand tout n'est pas dit, les principaux jalons sont, du moins, posés. Le troisième est indirect: par sa seule existence, cette étude suscite les questions, les mises au point, les recherches, comme en témoigne déjà la livraison de mars 1984 du Bulletin de la Bibliothèque nationale du Québec.

L'André Laurendeau de M. Monière est une oeuvre de pionnier. Du coup, il était inévitable qu'elle reste à parfaire en tel ou tel de ses aspects. Ayant été très proche d'André Laurendeau, je suis évidemment extrêmement sensible, peut-être trop, à toute inexactitude, même la plus minime. C'est pourquoi, en guise de conclusion, je me bornerai à répéter que le portrait que $M$. Monière a tracé de mon père est, dans l'ensemble, ressemblant. J'ai cru devoir le retoucher, mais je n'ai pas cru, je ne crois pas, qu'il faille le refaire.

\section{Corrections}

Le livre de M. Monière constitue d'ores et déjà la principale source d'information sur la carrière d'André Laurendeau. Me permettra-t-on donc, en terminant, de signaler à l'attention des lecteurs et des chercheurs quelques erreurs de fait ou de date échappées à la vigilance de M. Monière?

Blanche Hardy n'était pas «professeure de chant» (p. 16 et 17), mais une pianiste, qui enseignait aussi le solfège et le piano. Le professeur de chant, c'était Arthur Laurendeau, son mari. C'est en tant qu'accompagnatrice, donc, qu'elle fit avec Sarah Fischer le voyage à Londres.

Au collège Sainte-Marie, dont M. Monière nous offre un bien noir tableau, André Laurendeau ne fut pas «interne» (p. 23), mais externe. À cette époque, il habitait d'ailleurs tout près du collège, au 66 de la rue Hutchison (plus tard renuméroté 3472, aujourd'hui démoli). (p. 32).

Le pianiste Léo-Pol Morin n'a jamais écrit son prénom «Léopold»

Presque partout, ma mère et sa famille, des Perrault, sont transformés à tort en «Perreault» (p. 33, 38-41, 74, 75, 79, 170, 173 et 186).

Clément Latour était le nom de théâtre de Bernard Hogue. Il n'y avait donc qu'une personne là où $\mathrm{M}$. Monière en compte deux (p. 33).

«Jeune-Canada» est presque partout orthographié «Jeunes-Canada» (p. 53-55, 58, 59, 61-65, 67, 69-73, 75, 76, 79, 98, 100, 108, 127, 194 et 346). André Laurendeau écrivait pourtant avec constance les Jeune-Canada; et c'est l'orthographe qu'on trouve dans toutes les publications du mouvement. Ne surtout pas dire les «Jeunes-Cana- 
diens» (p. 88)! Il faut écrire, de même, Jeune-France, non «JeunesFrance» (p. 54).

M. Thuribe Belzile, qui n'avait rien fait pour mériter un prénom pareil, se voit ici affublé de celui de «Thurbide» (p. 54, 71, 73 et 76).

Dans la longue citation de la page 115 , à la troisième ligne en partant de la fin, on devrait trouver non pas «surtout après l'échec de la Belgique, de la Tchécoslovaquie, de la Pologne», mais: «surtout depuis Napoléon, Paris a appauvri et parfois broyé la province.»

Aux élections provinciales de 1939, les libéraux ont remporté soixante-dix sièges, non «64» (p. 133).

M. Monière parle par erreur, à propos du procès Chaloult, d'une intervention du «procureur général de la province, Gaspard Fauteux» (p. 150). À cette époque, le procureur général était Léon Casgrain, non Gaspard Fauteux qui n'a jamais exercé cette fonction. De toute façon, il s'agit ici, non pas du procureur général, mais de son premier substitut, $\mathrm{M}^{\mathrm{e}}$ Gérald Fauteux, qui agissait suivant les instructions du ministère de la Justice fédérale, dont le titulaire était Louis Saint-Laurent ${ }^{31}$.

Aux élections provinciales de 1944, l'Union nationale a remporté quarante-sept sièges (quarante-huit, si on inclut celui de Charlevoix obtenu quelques semaines plus tard), non «46» (p. 164).

La photographie de la page 172 ne date pas de juin 1935. Elle a été prise pas le photographe Albert Dumas vers 1945. (André Laurendeau n'a pris qu'en 1943 ou en 1944 le parti de porter la moustache, pour se donner l'air moins jeune et faire un chef de parti plus «présentable».) Les photographies des pages 173 et 174 datent de 1944.

Au moment où il devenait rédacteur au Devoir, André Laurendeau avait trente-cinq ans, non «36» (p. 205).

Vérification faite auprès du service Documentation - dossiers de Radio-Canada, l'émission Pays et merveilles a duré de décembre 1952 à juin 1961, non jusqu'en «1959» (p. 218).

Les Voyages au pays de l'enfance ne sont pas un «roman» (p. 13), dont ils n'ont pas du tout l'unité, et André Laurendeau n'avait pas précisément entrepris d'y «rassembler ses souvenirs d'enfance» (p. 245). Il s'agit plutôt de courts récits et d'impressions inspirés à la fois de certains souvenirs d'enfance et du spectacle de ses propres enfants.

André Laurendeau n'a pas écrit «les textes» des «quinze émissions» de la série Affaires de famille; ce n'est pas lui, non plus, qui y «analysait les comportements du couple à la lumière des facteurs socio-

\footnotetext{
31 Cf. André Laurendeau, La crise de la conscription (1942), 131, et René Chaloult, Mémoires politiques (Montréal, Éditions du Jour, 1969), 150.
} 
économiques» (p. 250). La série a comporté une quarantaine d'émissions, qui ont été diffusées durant l'année 1959-1960. Chaque émission comprenait, à peu près pour moitié, un sketch illustrant un aspect de la vie de couple ou de famille, et un commentaire, par un psychologue ou autre spécialiste, des problèmes illustrés par le sketch. André Laurendeau a écrit dix-huit ou dix-neuf de ces sketches, et Marcel Dubé et Pauline Lamy, les autres.

Page 254, l'auteur attribue à Réal Benoit des propos qui sont d'André Laurendeau et qui, contrairement à ce qu'il croit, ne concernent ni Guillaume - personnage assez central de Marie-Emma - ni sa famille, mais le frère Onésiphore - personnage tout à fait secondaire - et sa communauté religieuse. On ne peut donc strictement rien en tirer quant au sens global de ce téléthéâtre. Cela ne nous éclaire que sur la crainte qu'avait André Laurendeau qu'on lui fasse reproche d'avoir caricaturé un religieux ${ }^{32}$.

M. Monière note (p. 257) les réactions défavorables de la plupart des critiques devant les représentations de Deux femmes terribles. Il me paraît opportun de signaler que Laurendeau en fut probablement d'autant plus meurtri qu'il avait eu un bon motif pour espérer un accueil plus chaleureux. Il avait, en effet, d'abord présenté sa pièce sous l'anonymat au troisième concours du Théâtre du Nouveau-Monde, en 1960. C'est à l'unanimité qu'un jury composé, entre autres, de Guy Boulizon, Marcel Dubé et Gilles Hénault, lui avait décerné le prix.

M. Monière m'en voudra-t-il si j'avoue, pour clore ce chapitre, être réfractaire à des expressions comme «différence costructurante» (p. 13), «contemplation déréalisante» (p. 107) ou «poids de sa socialisation» (p. 282), que je ne suis pas du tout certain d'avoir comprises?

32 La référence n'est pas bonne non plus. La citation provient de la page 92 , non de la «p. 91» (p. 259, n. 23). 